\title{
Incremental Convex Hull Algorithms Are Not Output Sensitive*
}

\author{
D. Bremner \\ Department of Mathematics, University of Washington, \\ Box 354350, Seattle, WA 98195-4350, USA \\ bremner@math.washington.edu
}

\begin{abstract}
A polytope is the bounded intersection of a finite set of half-spaces of $\mathbb{R}^{d}$. Every polytope can also be represented as the convex hull conv $\mathcal{V}$ of its vertices (or extreme points) $\mathcal{V}$. The convex hull problem is to convert from the vertex representation to the halfspace representation or (equivalently by geometric duality) vice versa. Given an ordering $v_{1} \cdots v_{n}$ of the input vertices, after some initialization an incremental convex hull algorithm constructs half-space descriptions $\mathcal{H}_{n-k} \cdots \mathcal{H}_{n}$ where $\mathcal{H}_{i}$ is the half-space description of $\operatorname{conv}\left\{v_{1} \cdots v_{i}\right\}$. Let $m_{i}$ denote $\left|\mathcal{H}_{i}\right|$, and let $m$ denote $m_{n}$. Let $\varphi(d)$ denote $d /\lceil\sqrt{d}\rceil-1$; in this paper we give families of polytopes for which $m_{n-1} \in \Omega\left(m^{\varphi(d)}\right)$ for any ordering of the input. We also give a family of 0/1-polytopes with a similar blowup in intermediate size. Since $m_{n-1}$ is not bounded by any polynomial in $m, n$, and $d$, incremental convex hull algorithms cannot in any reasonable sense be considered output sensitive. It turns out that the same families of polytopes are also hard for the other main types of convex hull algorithms known.
\end{abstract}

\section{Introduction}

A polytope is the bounded intersection of a finite set of half-spaces of $\mathbb{R}^{d}$. A fundamental theorem of convexity is that every polytope can be represented as the convex hull of its extreme points or vertices. Converting from the vertex representation to the half-space representation is called facet enumeration. Converting from the half-space representation to the vertex representation is called vertex enumeration. Since these problems are equivalent under point-hyperplane duality, where the distinction is unimportant we use the term convex hull problem to refer to either or both problems.

The term output sensitive is used to describe algorithms with performance guarantees

\footnotetext{
* This research was supported by FCAR Québec and NSERC Canada. A preliminary version appeared in Proceedings of ISAAC '96, Lecture Notes in Computer Science, Springer-Verlag, New York. This work was done while the author was a student in the School of Computer Science at McGill University.
} 
in terms of the output size as well as the input size. For problems such as the convex hull problem, where the output size can range from exponential to logarithmic in the input size, such a bound is highly desirable. Implicit in describing an algorithm as output sensitive is that the dependence on the output size is "reasonable," usually bounded by a small polynomial. Here we accept any polynomial bound. For a $d$-dimensional polytope (or $d$-polytope) $P$ with $n$ vertices and $m$ facets we define the summed input and output size as size $P=(m+n) d$. It is an open problem whether there is a convex hull algorithm polynomial in size $P$. In the rest of this paper we call such an algorithm polynomial, where the dependence on size $P$ is implicit.

The only known class of algorithms not previously known to be superpolynomial [12], [3], or to have an NP-complete subproblem [15] are incremental algorithms based on the double description method of Motzkin et al. [22]. In this paper we show that any incremental algorithm is superpolynomial in the worst case.

Geometric algorithms in high dimensions are sometimes analyzed under the assumption that the number of input objects grows much faster than the dimension $d$. In this context, $d$ is considered a constant for the purposes of analysis. The algorithm of Chazelle [9] has an upper bound of $O\left(n^{\lfloor d / 2\rfloor}\right)$ in each fixed dimension $d>3$ and is thus worst-case optimal, up to factors that depend only on $d$. With $d$ fixed, a satisfactory output-sensitive time bound may be $O\left((m+n)^{c}\right)$ for some $c$ independent of the dimension (i.e., factors superpolynomial in the dimension may be acceptable). We show here that even this weaker condition cannot be met by incremental algorithms.

Convex hull computations are one area where assumptions of general position or nondegeneracy seem to make a great deal of difference to the tractability of the problem. While vertex enumeration algorithms based on pivoting that are polynomial on simple input have been known for over forty years (see [8], and refinements in, for example, [25], [24], [4], and [7]), finding an algorithm polynomial for nonsimple (so-called degenerate) input has proved a much more difficult undertaking. The (unmodified) pivoting method searches all feasible bases (sets of $d$ half-spaces that define a vertex) of the polytope. In general the number of feasible bases can be superpolynomial in size $P$. Neither of the two well-known modifications-perturbation and recursion on the dimension-yields a polynomial algorithm (see [3]).

Incremental algorithms are not necessarily affected by degeneracy, but have a fundamental weakness of their own. In an incremental facet enumeration algorithm, after some initialization, we insert points one by one, maintaining the convex hull of the points inserted so far at every step. A necessary condition for such algorithms to be polynomial is that the size of each intermediate polytope be polynomial in size $P$. It turns out the order the points are inserted can make a huge difference in the size of the intermediate polytopes. This is analogous to the simplex method of linear programming where a family such as the Klee-Minty [19] cubes can be superpolynomial for one pivoting rule but easily solvable using a different pivoting rule. Dyer [12] gave a family of polytopes for which inserting the points in the order given yields superpolynomial intermediate polytopes. Avis et al. [3] showed that several more sophisticated insertion orders used in practice also produce superpolynomial intermediate polytopes in the worst case. In this paper, we show that there are families for which there is no polynomial insertion order. This may be contrasted with the situation of the simplex method, where the existence of a polynomial pivoting rule remains an open problem. 


\section{Preliminaries}

In this section we introduce some notation and fundamental results from the theory of convex polytopes that will be useful in the sequel. We also make precise our measures of complexity.

Given a set of points $X=\left\{x_{1} \cdots x_{n}\right\}$, a combination $\sum_{i=1}^{n} \lambda_{i} x_{i}$ is called affine if $\sum \lambda_{i}=1$. An affine combination is called convex if every $\lambda_{i}$ is nonnegative. The affine hull aff $X$ is the set of all affine combinations of $X$, or equivalently the smallest affine subspace containing $X$. The dimension $\operatorname{dim} X$ is the dimension of aff $X$. The convex hull conv $X$ is the set of all convex combinations of $X$. Point $p$ is extreme for $X$ if $p$ is not a convex combination of $X \backslash\{p\}$.

Where convenient, we treat sets of points and matrices interchangeably, where the points correspond to rows of the matrix. We use $1\left(\mathbb{1}^{k}\right)$ for a vector of all ones (of length $k$ ) and $\mathrm{O}\left(\mathrm{O}^{k}\right)$ for a vector of all zeros (i.e., the origin).

A hyperplane $h$ supports a polytope $P$ if $h \cap P \neq \emptyset$ and $P$ is contained in one of the closed half-spaces (the supporting half-space) induced by $h$. The faces of $P$ are $\emptyset, P$, and the intersection of $P$ and a supporting hyperplane of $P$. Faces of dimension $k$ are called $k$-faces; $f_{k}(P)$ denotes the number of $k$-faces of $P$. The names vertices, edges, and facets refer to 0,1 , and $(d-1)$-faces, respectively. For a facet $F$ with supporting hyperplane $h$, we write $F^{+}$for the corresponding supporting half-space and $F^{-}$for the other (open) half-space induced by $h$. The face lattice of a polytope is the poset of its faces partially ordered by inclusion. Polytopes $P$ and $Q$ are combinatorially equivalent (resp., dual) if their face lattices are isomorphic (resp., anti-isomorphic, i.e., isomorphic with the direction of inclusion reversed). For any point set $X$, the polar $X^{*}$ of $X$ is defined as $\{y \mid X y \leq 1\}$. It is known (see, e.g., [5]) that if $P$ is a polytope containing the origin in its interior, $P^{*}$ is a polytope dual to $P$, containing the origin in its interior.

A family of polytopes is used here to mean an infinite set of polytopes. Usually, but not necessarily, families arise in some natural way from a problem such as the Traveling Salesman Problem [13], or a construction such as those described below. Given a family of polytopes $\mathcal{F}$, a function $g: \mathcal{F} \rightarrow \mathbb{R}$ is called polynomial for $\mathcal{F}$ if there exists some univariate polynomial $p(x)$ such that for every $P \in \mathcal{F}, g(P) \leq p($ size $P)$. A function $g: \mathcal{F} \rightarrow \mathbb{R}$ is called weakly polynomial for $\mathcal{F}$ if there exists positive function $f(x)$ and polynomial $p(x)$ such that $\forall P \in \mathcal{F}, g(P) \leq f(\operatorname{dim} P) \cdot p($ size $P)$; this corresponds to the notion of considering $d$ to be a constant. If $g$ is not (weakly) polynomial for $\mathcal{F}$ we say that $g$ is (strongly) superpolynomial for $\mathcal{F}$.

The convex hull of $n$ points on the moment curve $c(t)=\left(t, t^{2}, \ldots, t^{d}\right)$ is called the cyclic polytope on $n$ vertices, written here as $C_{d}(n)$. Here we assume that the $n$ vertices are $\{c(0), c(1), \ldots, c(n-1)\}$. Let $\gamma(n, d)$ denote the number of facets of $C_{d}(n)$. It follows from "Gale's evenness condition" [17], [18], [27] that:

$$
\gamma(n, d)=\left\{\begin{array}{cc}
\frac{n}{n-d / 2}\left(\begin{array}{c}
n-d / 2 \\
n-d
\end{array}\right), & d \text { even} ; \\
2\left(\begin{array}{c}
n-(d+1) / 2 \\
n-d
\end{array}\right), & d \text { odd }
\end{array}\right.
$$


The famous Upper Bound Theorem of McMullen [21] says that no $d$-polytope with $n$ vertices has more than $\gamma(n, d)$ facets.

A very useful construction for building new "more complicated" families of polytopes from known families is the Cartesian product of polytopes construction. Let $P$ be a subset of $\mathbb{R}^{k}$ and let $Q$ be a subset of $\mathbb{R}^{l}$. Let $P \times Q$, called the product of $P$ and $Q$, denote $\{(p, q) \mid p \in P, q \in Q\}$. We regard $\mathbb{R}^{l} \times \mathbb{R}^{k}$ as naturally embedded in $\mathbb{R}^{k+l}$. The following lemma (whose proof we omit) summarizes the basic properties of the construction:

Lemma 1. Let $P$ be a k-polytope and $Q$ an l-polytope.

(a) $P \times Q$ is a $(k+l)$-polytope.

(b) For $i \geq j \geq 0$, the $i$-faces of $P \times Q$ are precisely $F_{p} \times F_{q}$ where $F_{p}$ is a $j$-face of $P$ and $F_{q}$ is an $(i-j)$ face of $Q$.

(c) $P \times Q$ has $f_{0}(P) \cdot f_{0}(Q)$ vertices and $f_{k-1}(P)+f_{l-1}(Q)$ facets.

For polytopes $P \subset \mathbb{R}^{k}$ and $Q \subset \mathbb{R}^{l}$, we define the orthogonal sum $P \oplus Q$ as

$$
P \oplus Q \equiv \operatorname{conv}\left(\left\{\left(p, \mathrm{O}^{l}\right) \mid p \in P\right\} \cup\left\{\left(\mathrm{O}^{k}, q\right) \mid q \in Q\right\}\right) .
$$

Suppose $P$ and $Q$ are $k$ and $l$ polytopes, respectively, that contain the origin as an interior point. By Lemma 1 and polarity, we know the defining half-spaces for $P^{*} \times Q^{*}$ can be written as

$$
\left[\begin{array}{cc}
\mathcal{V}(P) & 0 \\
O & \mathcal{V}(Q)
\end{array}\right] x \leq 1
$$

It follows that

$$
P \oplus Q=\left(P^{*} \times Q^{*}\right)^{*} .
$$

In particular, this means that $P \oplus Q$ has $f_{0}(P)+f_{0}(Q)$ vertices and $f_{k-1}(P) \cdot f_{l-1}(Q)$ facets.

\section{Polytopes Without Good Insertion Orders}

In the most general sense, insertion orders are procedures to determine at each step of an incremental algorithm, what input element should be processed next. In some cases, such as lexicographic or random ordering, all of the choices can be made before the input is processed. In other cases, such as the maxcutoff rule (where we choose the next element which causes the largest drop in intermediate size), the insertion order is inherently dynamic. In either case, for every input and for every insertion order there are one or more possible permutations of the input generated. We will say that $\pi$ is a good insertion order for $\mathcal{F}$ if the size of intermediate polytopes created by $\pi$ is polynomial for $\mathcal{F}$ (obviously a much stronger bound is necessary for an insertion order to be "good" in practice). A good insertion order does not by itself guarantee a polynomial algorithm: in particular, the use of triangulation can still cause an incremental algorithm to be superpolynomial (see [3]). On the other hand, a naive implementation of the double 
description method will be polynomial given a good insertion order. We show that there are polytopes for which every permutation is bad; in fact we show the slightly stronger result that every permutation is bad at the last step.

\subsection{The Main Result}

At each step of an incremental facet enumeration algorithm, we maintain (at minimum) the vertex description $\mathcal{V}_{i}$ and the half-space description $\mathcal{H}_{i}$ of the current intermediate polytope (the "double description" of Motzkin et al. [22]). We are interested here in the drop in the size of the half-space description caused by inserting the last vertex. This is the same as the increase in the number of facets caused by removing one vertex of a polytope and recomputing the convex hull of the remaining vertices. In the following definitions, let $P$ be a $d$-polytope and let $v$ be a vertex of $P$. Let $\mathcal{V}(P)$ denote the vertices of $P$. Let $P \ominus v$ denote $\operatorname{conv}(\mathcal{V}(P) \backslash\{v\})$. Let $\mathcal{F}(P)$ denote the facets of $P, \mathcal{F}_{v}(P)$ the facets of $P$ containing $v$, and let $\widetilde{\mathcal{F}}_{v}(P)$ be defined as follows:

$$
\widetilde{\mathcal{F}}_{v}(P) \equiv \begin{cases}\left\{F \in \mathcal{F}(P \ominus v) \mid v \in F^{-}\right\} & \text {if } \operatorname{dim}(P \ominus v)=d, \\ \{P \ominus v\} & \text { otherwise. }\end{cases}
$$

We define $\operatorname{loss}(v, P) \equiv\left|\widetilde{\mathcal{F}}_{v}(P)\right|$ as the number of half-spaces deleted by inserting $v$ last (we make the notation simplifying assumption that in the case where $\operatorname{dim}(P \ominus v)=$ $\operatorname{dim}(P)-1$, the affine hull of $P \ominus v$ is stored as two half-spaces). Similarly, we define gain $(v, P) \equiv\left|\mathcal{F}_{v}(P)\right|$ as the number of half-spaces created by inserting $v$ last. The net drop in intermediate size is then $\operatorname{drop}(v, P)=\operatorname{loss}(v, P)-$ gain $(v, P)$. Finally we define $\operatorname{gain}(P) \equiv \max _{v} \operatorname{gain}(v, P), \operatorname{loss}(P) \equiv \min _{v} \operatorname{loss}(v, P)$, and $\operatorname{drop}(P) \equiv$ $\min _{v} \operatorname{drop}(v, P) \cdot \operatorname{drop}(P)$ could be negative if there is a vertex whose removal decreases the number of facets (as in, for example, a stacked polytope).

The lower bounds in this paper follow from using the product and sum of polytopes constructions defined above. The central geometric observation is that while the number of facets of the final polytope sums under the product of polytopes operation, the number of intermediate facets multiplies. We call a polytope $P$ robust if $\operatorname{dim}(P \ominus v)=\operatorname{dim} P$ for every vertex $v$ of $P$.

Theorem 1. Let $P$ and $Q$ be polytopes with dimension at least $2 . P \times Q$ is robust and

$$
\operatorname{drop}(P \times Q)=\operatorname{loss}(P) \cdot \operatorname{loss}(Q) .
$$

We prove Theorem 1 via several lemmas. Since gain $(v, P)$ is nonnegative, the following holds:

$$
\operatorname{loss}(P)-\operatorname{gain}(P) \leq \operatorname{drop}(P) \leq \operatorname{loss}(P) .
$$

Let $P$ and $Q$ be polytopes with dimension at least 2 . Theorem 1 follows from the following two facts:

$$
\begin{aligned}
\operatorname{gain}(P \times Q) & =0, \\
\operatorname{loss}(P \times Q) & =\operatorname{loss}(P) \cdot \operatorname{loss}(Q) .
\end{aligned}
$$


We start with (4), which is equivalent to saying that every facet of $(P \times Q)$ is robust. Since each facet of $P \times Q$ is the product of a facet of $P$ (resp., $P$ ) and $Q$ (resp., a facet of $Q$ ), this follows from the next lemma:

Lemma 2. Let $P$ and $Q$ be polytopes with $\operatorname{dim} P \geq 1$ and $\operatorname{dim} Q \geq 1 . P \times Q$ is robust.

Proof. Let $v=(p, q)$ be a vertex of $P \times Q$. Let $P^{\prime}$ denote $(P \times Q) \ominus v$ :

$$
P^{\prime}=\operatorname{conv}(\{(P \ominus p) \times Q\} \cup\{P \times(Q \ominus q)\}) .
$$

If $\operatorname{dim}(Q \ominus q)=\operatorname{dim} Q$, then the lemma follows by Lemma 1. Otherwise, $q \notin \operatorname{aff}(Q \ominus q)$. Let $p^{\prime}$ be some vertex of $P$ other than $p$. If $(x, y) \in \operatorname{aff}(X \times Y)$, by manipulation of sums we see that $x \in$ aff $X$ and $y \in \operatorname{aff} Y$. It follows that $\left(p^{\prime}, q\right) \notin \operatorname{aff}(P \times(Q \ominus q))$. But $\left(p^{\prime}, q\right) \in P^{\prime}$, so

$$
\operatorname{dim} P^{\prime}>\operatorname{dim}[P \times(Q \ominus q)] \geq \operatorname{dim} P+\operatorname{dim} Q-1 .
$$

We now turn our attention to (5).

Lemma 3. Let $P$ and $Q$ be polytopes containing the origin as a vertex.

$$
\widetilde{\mathcal{F}}_{\mathrm{O}}(P \times Q)=\left\{F_{p} \oplus F_{q} \mid F_{p} \in \widetilde{\mathcal{F}}_{\mathrm{O}}(P), F_{q} \in \widetilde{\mathcal{F}}_{\mathrm{O}}(Q)\right\} .
$$

Proof. Let $P^{\prime}$ denote $(P \times Q) \ominus$ O. Suppose we have facets $F_{p} \in \widetilde{\mathcal{F}}_{\mathrm{O}}(P), F_{q} \in \widetilde{\mathcal{F}}_{\mathrm{O}}(Q)$. We can write linear constraints $a x \geq 1$ and $b y \geq 1$ that support $P^{\prime}$ in $F_{p}$ and $F_{q}$, respectively. The constraint $a x+b y \geq 1$ supports $P^{\prime}$. The vertices of $P \times Q$ that lie on $a x+b y=1$ are precisely the vertices of $F_{p} \oplus F_{q}$. It is known (see, e.g., [23]) that if aff $P_{1} \cap$ aff $P_{2}=\emptyset$ and $P_{1}$ is not a translate of $P_{2}$, then $\operatorname{dim}\left(P_{1} \oplus P_{2}\right)=\operatorname{dim} P_{1}+$ $\operatorname{dim} P_{2}+1$.

Now suppose we have some $F \in \widetilde{\mathcal{F}}_{\mathrm{O}}(P \times Q)$. Let $h$ denote aff $F$. Every vertex of $P \times Q$ in $h$ must have at least one adjacent edge $e$ intersecting $F^{-}$, since otherwise $P \times Q \subset F^{+}$. The other vertex of $e$ must be $\mathrm{O}$, since otherwise $h$ is not a supporting hyperplane for $(P \times Q) \ominus \bigcirc$. By Lemma 1 the vertices defining $h$ must be of the form $(p, \bigcirc)$ or $(\mathrm{O}, q)$ for $p \in \mathcal{V}(P), q \in \mathcal{V}(Q)$. It follows that any basis (set of $d$ affinely independent vertices) $B$ defining $h$ must have the form

$$
B=\left[\begin{array}{cc}
B_{p} & \bigcirc \\
\bigcirc & B_{q}
\end{array}\right],
$$

where $B_{p}\left(\right.$ resp., $\left.B_{q}\right)$ is a basis of some $F_{p} \in \widetilde{\mathcal{F}}_{\mathrm{O}}(P)\left(\operatorname{resp} ., F_{q} \in \widetilde{\mathcal{F}}_{\mathrm{O}}(Q)\right)$.

Since by change of coordinates we can assume without loss of generality that an arbitrary vertex of $P \times Q$ lies on the origin, (5) and hence Theorem 1 follows. From Lemma 3 we also get a complete characterization of the facets of $(P \times Q) \ominus v$ since

$$
\mathcal{F}((P \times Q) \ominus v)=\left\{F \in \mathcal{F}(P \times Q) \mid v \in F^{+}\right\} \cup \widetilde{\mathcal{F}}_{v}(P \times Q) .
$$




\subsection{Consequences}

We now present some consequences of Theorem 1 for particular families of polytopes. To construct families of polytopes hard for incremental convex hull algorithms, it suffices to take products of families with large loss functions. In this subsection we present three such families. Recall that $C_{d}(n)$ denotes the cyclic $d$-polytope with $n$ vertices.

Lemma 4. For even $d, n \geq d+2$, for any vertex $v$ of $C_{d}(n)$,

$$
\begin{aligned}
\operatorname{loss}\left(v, C_{d}(n)\right) & =\left(\begin{array}{c}
n-d / 2-2 \\
n-d-1
\end{array}\right)=\left[\frac{d(n-d)}{(2 n-d-2) n}\right] \gamma(n, d) \\
& \in \Theta\left(n^{d / 2-1}\right), \quad \text { d fixed. }
\end{aligned}
$$

Proof. Let the dimension $d=2 k$ for some positive integer $k$. Let $v$ be a vertex of $P=C_{d}(n)$. It is known (see, e.g., [2, p. 102]) that each vertex of an even-dimensional cyclic polytope is contained in $\gamma(n-1, d-1)$ facets. Since $P$ is $\operatorname{simplicial}$, gain $(v, P)=$ $\gamma(n-1, d-1)$. Since $P \ominus v$ is full dimensional, $f_{d-1}(P \ominus v)-f_{d-1}(P)=\operatorname{loss}(v, P)-$ gain $(v, P)$. It follows that

$$
\begin{aligned}
\operatorname{loss}(v, P) & =f_{d-1}(P \ominus v)-f_{d-1}(P)+\operatorname{gain}(v, P) \\
& =\gamma(n-1, d)-\gamma(n, d)+\gamma(n-1, d-1) .
\end{aligned}
$$

Substituting in the appropriate values of $\gamma(n, d)$ from (1) for odd and even $d$

$$
\begin{aligned}
\operatorname{loss}(v, P) & =\left[\frac{n-1}{k}-\frac{(n-k-1) n}{(n-2 k) k}+\frac{2 n-2 k-2}{n-2 k}\right] \frac{(n-k-2) !}{(n-2 k-1) !(k-1) !} \\
& =\frac{(n-k-2) !}{(n-2 k-1) !(k-1) !}
\end{aligned}
$$

A polytope $P$ is called centered if for every $v \in \mathcal{V}(P), P \ominus v$ contains the origin as an interior point. We describe here a family of centered convex polygons $P_{n}$ with integer coordinates bounded by $O\left(n^{2}\right)$. This will allow us to construct a family of polytopes with an asymptotic loss function similar to that of cyclic polytopes, but much better numerical behavior. For odd $n$, choose points as follows:

$$
\begin{aligned}
& x_{i}=(n-1) / 2-i, \\
& y_{i}=2 x_{i}^{2}-3,
\end{aligned} \quad i=0, \ldots, n-1 .
$$

If $n$ is even, construct $P_{n-1}$ and then add a vertex with coordinates $\left(0, n^{2} / 2-2 n\right)$. Figure 1 illustrates $P_{6}$. The reader can verify that $P_{5}$ is centered. Since $\mathcal{V}\left(P_{5}\right) \subset \mathcal{V}\left(P_{n}\right)$ for $n>5, P_{n}$ is centered for $n \geq 5$.

The following lemma gives a general method for constructing families of polytopes with large loss functions:

Lemma 5. Let $P$ be a centered polytope. Let $Q$ be a polytope with $m$ facets containing the origin in its interior. Let $v=(p, O)$ be a vertex of $P \oplus Q$,

$$
\operatorname{loss}(v, P \oplus Q)=\operatorname{loss}(p, P) \cdot m \text {. }
$$




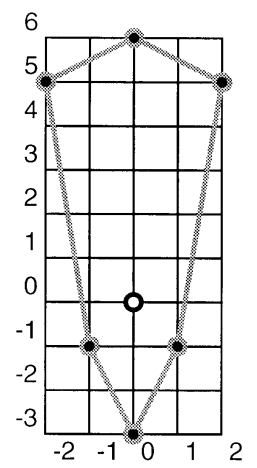

Fig. 1. $P_{6}$.

Proof. $\quad$ Note that $(P \oplus Q) \ominus(p, O)=(P \ominus p) \oplus Q$. Since $P$ is centered, by Lemma 1 and polarity, $a x+b y \leq 1$ defines a facet of $(P \ominus p) \oplus Q$ iff $a x \leq 1$ defines a facet of $P \ominus p$ and $b y \leq 1$ defines a facet of $Q$. Vertex $(p, O)$ is infeasible for $a x+b y \leq 1$ iff $p$ is infeasible for $a x \leq 1$.

For a polytope $P$, let $\bigoplus_{k} P$ denote the $k$-fold sum of $P$ with itself, i.e., $\bigoplus_{1} P \equiv P$, $\bigoplus_{k} P \equiv P \oplus \bigoplus_{k-1} P$. Let $P_{n}$ be a centered convex polygon with $n$ vertices. Let $\Pi_{d}(n)$ denote $\bigoplus_{d / 2} P_{n}$.

Corollary 1. For even $d, n \geq 5$, loss $\Pi_{d}(n)=n^{d / 2-1}$.

From Lemma 4 and Corollary 1, we have two families of polytopes with large loss functions. Intuitively, our construction takes the $\sqrt{d}$-fold product of $\sqrt{d}$-polytopes from these families. In order to have example polytopes in each sufficiently large dimension, a slightly more complex construction is necessary. Following [3], for any even dimension $2 d \geq 4$ define $a=\lceil\sqrt{d}\rceil, b=\lfloor d / a\rfloor, c=d \bmod a$, and $K_{2 d}(n)=Q_{2 a}^{b}(n) \times Q_{2 c}(n)$ where $Q_{k}(n)$ is either a cyclic polytope $C_{k}(n)$ or a sum of polygons $\Pi_{k}(n)$.

Theorem 2. For $d \geq 2$ held fixed:

(a) $s \equiv \operatorname{size} K_{2 d}(n) \in O\left(n^{\lceil\sqrt{d}\rceil}\right)$; and

(b) $\operatorname{drop} K_{2 d}(n) \in \Omega\left(s^{d /\lceil\sqrt{d}\rceil-1}\right)$.

Proof. Let $\beta=\lceil d / a\rceil$. We will make use of the fact that $\beta \leq a$. By Lemma $1, K_{2 d}(n)$ has $\Theta\left(n^{\beta}\right)$ vertices and $\Theta\left(n^{a}\right)$ facets. It follows that size $K_{2 d}(n) \in \Theta\left(n^{a}\right)$. By Theorem 1 , $\operatorname{drop} K_{2 d}(n) \in \Omega\left(n^{\varphi(d)}\right)$ where

$$
\begin{aligned}
\varphi(d) & = \begin{cases}(a-1) b & \text { if } d \bmod a=0, \\
(a-1) b+(c-1) & \text { otherwise, }\end{cases} \\
& =d-\beta \\
& =a(d / a-\beta / a) \\
& \geq a(d / a-1) .
\end{aligned}
$$


From this theorem we can see that incremental convex hull algorithms are strongly superpolynomial in the worst case, irrespective of the insertion order used. Since these same families have previously been shown to be hard for perturbation/triangulation and for face lattice producing algorithms, it follows that no method consisting of running several of the well-known methods in parallel will be output sensitive either.

An important class of polytopes for facet enumeration is the 0/1-polytopes, whose vertices are a subset of $\{0,1\}^{d}$. Because of their importance in combinatorial optimization, a facet enumeration algorithm polynomial for 0/1-polytopes would be significant result; unfortunately, incremental algorithms fail here also, at least from a theoretical point of view. We consider the equivalent case of polytopes whose vertices are a subset of $\{+1,-1\}^{d}$. Given $k$-polytope $P$ and an $l$-polytope $Q$, define the diagonal sum $P \odot Q$ as

$$
P \odot Q \equiv \operatorname{conv}\left(\left\{\left(x,-x_{k} \rrbracket^{l}\right) \mid x \in P\right\} \cup\left\{\left(y_{1} \mathbb{1}^{k}, y\right) \mid y \in Q\right\}\right) .
$$

The idea behind the diagonal sum (a construction due to Kortenkamp et al. [20]) is that $P$ and $Q$ are embedded in the two subspaces

$$
\begin{aligned}
-x_{k}=x_{k+1}=\cdots x_{k+l}, \\
x_{1}=x_{2}=\cdots=x_{k}=x_{k+1},
\end{aligned}
$$

where the subspace defined by (6a) intersects the subspace defined by (6b) exactly in the point $\mathrm{O}$. It turns out that this is equivalent in a very strong sense to the orthogonal sum construction. Call two polytopes linearly equivalent if there is a linear transformation from one to the other.

Lemma 6. Let $P$ and $Q$ be polytopes containing the origin in their interior. $R=P \odot Q$ is linearly equivalent to $R^{\prime}=P^{\prime} \oplus Q^{\prime}$, where $P^{\prime}$ (resp., $Q^{\prime}$ ) is linearly equivalent to $P$ (resp., $Q$ ).

Proof. Let $k=\operatorname{dim} P, l=\operatorname{dim} Q$ and $d=k+l$. Let $L_{1}$ ( $L_{2}$, respectively) be the linear subspace defined by (6a), ((6b), respectively). Let $B_{1}$ denote a basis for $L_{1}$ (i.e., a set of $k$ linearly independent vectors in $\left.L_{1}\right)$. Let $B_{2}$ be a basis for $L_{2}$. Since $\operatorname{dim}\left(L_{1} \cup L_{2}\right)=d$, $B=B_{1} \cup B_{2}$ forms a basis for $\mathbb{R}^{d}$. The linear transformation $B^{-1}$ transforms $B$ to the identity matrix $I$. Let $R^{\prime}=R B^{-1}$ (i.e., the transformation $B^{-1}$ applied to $R$ ). Since $L_{1} \cap L_{2}=\mathrm{O}, B_{1}$ and $B_{2}$ are transformed to disjoint subsets (of rows) of $I$, hence $R^{\prime}$ has the desired form $P^{\prime} \oplus Q^{\prime}$ (possibly after reordering coordinates). By construction,

$$
\begin{aligned}
& \mathcal{V}(R)=\mathcal{V}\left(R^{\prime}\right) B=\left[\begin{array}{cc}
\mathcal{V}\left(P^{\prime}\right) & \bigcirc \\
\bigcirc & \mathcal{V}\left(Q^{\prime}\right)
\end{array}\right]\left[\begin{array}{l}
B_{1} \\
B_{2}
\end{array}\right], \\
& {\left[\begin{array}{cc}
X & -x_{i, k} \\
y_{i, 1} & Y
\end{array}\right]=\left[\begin{array}{l}
\mathcal{V}\left(P^{\prime}\right) B_{1} \\
\mathcal{V}\left(Q^{\prime}\right) B_{2}
\end{array}\right], }
\end{aligned}
$$

where $X=\mathcal{V}(P)$ and $Y=\mathcal{V}(Q)$. It follows that, e.g., $P=P^{\prime} \widehat{B}_{1}$, where $\widehat{B}_{1}$ denotes the first $k$ columns of $B_{1}$.

In particular, it follows that Lemma 5 also holds with the $\oplus$ operation replaced by $\odot$. Let $H_{d}$ denote the hypercube with vertices $\{+1,-1\}^{d}$. The reader can verify that $H_{d}$ 
is centered for $d \geq 3$. Define $\bigodot_{k} P$ analogously to $\bigoplus_{k} P$. For $d=k^{2}$, define $U_{3 d}$ as $\left(\bigodot_{k} H_{3}\right)^{k}$. Since $\operatorname{loss}\left(H_{d}\right)=1$, by Theorem 1 and Lemma 5 we have the following:

Theorem 3. Let $d=k^{2}$ for some integer $k$ :

(a) $s \equiv \operatorname{size}\left(U_{3 d}\right) \in O\left(d^{\sqrt{d}}\right)=O\left(2^{\sqrt{d} \log d}\right)$; and

(b) $\operatorname{drop} U_{3 d} \in \Omega\left(2^{d}\right)=\Omega\left(s^{\sqrt{d} / \log d}\right)$.

Since duality preserves the size of the face lattice, $U_{3 d}$ has face lattice size $3^{3 d}$, hence is also difficult for face lattice generating convex hull algorithms. In [3], the authors observe that if $P$ has $\bar{f}$ nonempty faces, then any triangulation of the boundary of $P$ contains at least $(\bar{f}-1) / 2^{\operatorname{dim} P}$ maximal simplices. It follows that the family $U_{3 d}$ is also difficult for algorithms based on triangulation and perturbation. By a construction similar to the one in Theorem 2 we can obtain families with members in every sufficiently large dimension with about the same behavior.

\section{Conclusions}

In a previous paper Avis et al. [3] showed that products of cyclic polytopes (and products of sums of polygons) form a family hard for algorithms based on triangulation, perturbation, and on computing the face lattice. Here we have extended their results to show they are also hard for incremental algorithms, regardless of insertion order. We mention, in closing, a minor extension of [3] with regard to algorithms that compute the face lattice. Swart [25] suggested computing the "abbreviated face lattice" as follows. Rather than recursively computing the entire face lattice, stop the recursion at the first simplicial face (i.e., a $k$-face with $k+1$ vertices) encountered. While this avoids counterexamples based on "large-dimensional" simplicial faces, any product of $d$-polytopes will have no simplicial faces of dimension larger than $d$. Thus the families given here are also hard for convex hull algorithms that compute the abbreviated face lattice.

The question of the practical usefulness of the double description method is hardly settled by the existence of families without good insertion orders. There have been many practical success stories using this technique (see, e.g., [6] and [16]) and its simplicity and "immunity" to degeneracy make it the method of choice for most implementors (see, e.g., [1], [10], [14], and [26]). Nonetheless, the results of this paper show that rather than searching for a universally good insertion order, a more fruitful approach for families that defeat the usual heuristics (and pivoting) is to try and use knowledge about the combinatorial structure of the family to compute a good insertion order. Remarkable success in this vein has recently been reported by Deza et al. [11].

\section{Acknowledgments}

The author would like to thank David Avis, Komei Fukuda, and Raimund Seidel for useful and interesting conversations about convex hulls and for comments on a previous 
version of this paper. The polytopes of Theorem 3 were suggested to the author by David Avis. Raimund Seidel reminded the author that products of sums of polygons could stand in for products of cyclic polytopes. Finally, the author would like to thank an anonymous referee for helpful suggestions and corrections.

\section{References}

1. D. Alevras, G. Cramer, and M. Padberg. DODEAL. <ftp://elib.zib-berlin.de/pub/ mathprog/polyth/dda>.

2. A. Altshuler and M. Perles. Quotient polytopes of cyclic polytopes. Part I: Structure and characterization. Israel J. Math., 36(2):97-125, 1980.

3. D. Avis, D. Bremner, and R. Seidel. How good are convex hull algorithms? Comput. Geom.: Theory Appl., 7(5-6), 1997.

4. D. Avis and K. Fukuda. A pivoting algorithm for convex hulls and vertex enumeration of arrangements and polyhedra. Discrete Comput. Geom., 8:295-313, 1992.

5. A. Brøndsted. Introduction to Convex Polytopes. Springer-Verlag, New York, 1981.

6. G. Ceder, G. Garbulsky, D. Avis, and K. Fukuda. Ground states of a ternary lattice model with nearest and next-nearest neighbor interactions. Phys. Rev. B, 49:1-7, 1994.

7. T. M. Chan. Output-sensitive results on convex hulls, extreme points, and related problems. In: Proc. 11th ACM Symp. Comp. Geom., pp. 10-19, 1995.

8. A. Charnes. The simplex method: Optimal set and degeneracy. In: An Introduction to Linear Programming, Lecture VI, pp. 62-70. Wiley, New York, 1953.

9. B. Chazelle. An optimal convex hull algorithm in any fixed dimension. Discrete Comput. Geom., 10:377409, 1993.

10. T. Christof and A. Loebel. Porta. March 1997. <http://www.iwr.uni-heidelberg.de/iwr/ comopt/soft/PORTA/readme.html>.

11. A. Deza, M. Deza, and K. Fukuda. On Skeletons, Diameters and Volumes of Metric Polyhedra. Technical Report, Laboratoire d'Informatique de l'Ecole Supérieure, January 1996. Lecture Notes in Computer Science. Springer-Verlag, New York. To appear.

12. M. Dyer. The complexity of vertex enumeration methods. Math. Oper. Res., 8(3):381-402, 1983.

13. R. Euler and H. Le Verge. Complete linear descriptions of small asymetric travelling salesman polytopes. Discrete Appl. Math., 62:193-208, 1995.

14. K. Fukuda. cdd+ Reference manual, version 0.73. ETHZ, Zurich, Switzerland. <ftp://ifor13. ethz.ch/pub/fukuda/cdd>.

15. K. Fukuda, T. M. Liebling, and F. Margot. Analysis of backtrack algorithms for listing all vertices and all faces of a convex polyhedron. Comput. Geom.: Theory Appl., 8:1-12, 1997.

16. K. Fukuda and A. Prodon. The Double Description Method Revisited. Lecture Notes in Computer Science, vol. 1120. Springer-Verlag, New York, 1996. To appear.

17. D. Gale. Neighborly and cyclic polytopes. Proc. 7th Symp. Pure Math. (V. Klee, Ed.). 1961.

18. B. Grünbaum. Convex Polytopes. Interscience, London, 1967.

19. V. Klee and G. Minty. How good is the simplex method? In Inequalities-III (O. Shisha, ed.), pp. 159-175. Academic Press, New York, 1972.

20. U. H. Kortenkamp, J. Richter-Gebert, A. Sarangarajan, and G. M. Ziegler. Extremal properties of 0/1polytopes. Discrete Comput. Geom., 4(16), 1997.

21. P. McMullen. The maximal number of faces of a convex polytope. Mathematika, 17:179-184, 1970.

22. T. S. Motzkin, H. Raiffa, G. Thompson, and R. M. Thrall. The double description method. In Contributions to the Theory of Games II (H. Kuhn and A. Tucker, eds.). Annals of Math. Studies, vol. 8, pp. 51-73. Princeton University Press, Princeton, 1953.

23. J. A. Murtha and E. R. Willard. Linear Algebra and Geometry. Holt Rinehart and Winston, New York, 1969. 
24. R. Seidel. Output-size sensitive algorithms for constructive problems in computational geometry. Ph.D. thesis, Department Computer Science, Cornell University, Ithaca, NY, 1986. Technical Report TR 86-784.

25. G. Swart. Finding the convex hull facet by facet. J. Algorithms, pp. 17-48, 1985.

26. D. K. Wilde. A library for doing polyhederal applications. Technical Report 785, IRISA, Campus Universitaire de Beaulieu, 35042 Rennes CEDEX France, 1993. <ftp: / ftp. irisa. fr/local/API>.

27. G. M. Ziegler. Lectures on Polytopes. Graduate Texts in Mathematics. Springer-Verlag, New York, 1994.

Received November 13, 1996, and in revised form April 28, 1997. 